\title{
Incidencia del tiempo de internación en la terapia neonatal sobre las interacciones madre-bebé de alto riesgo
}

\author{
The effects length of hospitalization in neonatal \\ therapy has on interactions between \\ mother and high-risk baby
}

\author{
Maria Paulina HAUSER ${ }^{1}$ \\ Teresita Ana MILÁN ${ }^{2}$ \\ María Cristina GONZÁLEZ ${ }^{3}$ \\ Alicia OIBERMAN ${ }^{4}$
}

\begin{abstract}
Resumen
Se consideran como bebés de alto riesgo a aquellos que requieren cuidados especiales, independientemente de su edad gestacional o su peso de nacimiento. Esto implica que las primeras interacciones madre-hijo tendrán lugar en una Unidad de Cuidados Intensivos Neonatales. Se presentan los resultados preliminares de una investigación, en la cual se aplicó la Escala de observación del vínculo madre-bebé internado en una Unidad de Cuidados Intensivos Neonatales, con el propósito de describir las interacciones madre-bebé. La muestra estuvo compuesta por 36 duplas y se realizaron 166 observaciones que se clasificaron de acuerdo a la puntuación obtenida en la Escala de Observaciones (Adecuadas, Poco adecuadas, Inadecuadas), la cual se ha relacionado con el tiempo de internación del bebé en la unidad de terapia neonatal, con la finalidad de investigar si existe una asociación entre estas variables. Conocer el modo en cómo se presentan las interacciones durante la internación permitiría realizar una detección temprana de las dificultades, con el fin de evitar que resulten en trastornos durante la infancia.
\end{abstract}

Palabras Ilave: Relaciones madre-hijo; Servicios de salud del niño; Tiempo de internación.

\footnotetext{
Abstract

High-risk babies require special cares regardless of their gestational age or birth weight. Due to this, the first motherbaby interactions will be developed in a Neonatal Intensive Care Unit. Preliminary results of an investigation are

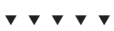

${ }^{1}$ Universidad Nacional de San Luis, Facultad de Psicología, Consejo Nacional de Investigaciones Científicas y Técnicas. Ejército de Los Andes 950, D5700HHW, Box 33, San Luis, Argentina. Dirección de correspondencia/Correspondence to: M.P. HAUSER. E-mail: <paulinahauser@hotmail.com>.

2 Universidad Nacional de San Luis, Facultad de Psicología. San Luis, Argentina.

3 Universidad Nacional de Córdoba, Facultad de Psicología. Córdoba, Argentina.

${ }^{4}$ Universidad de Buenos Aires, Facultad de Psicología, Consejo Nacional de Investigaciones Científicas y Técnicas. Buenos Aires, Argentina. Agradecimientos: Se agradece al Consejo Nacional de Investigaciones Científicas y Técnicas y al Servicio de Neonatología de la Maternidad Provincial "Dra. Teresita Baigorria", Provincia de San Luis, Argentina.
} 
presented, and for this study an Observational Scale of the bond between mother and baby in the Neonatal Intensive Care Unit was applied for the purpose of describing these mother-baby interactions. The sample was composed of 36 pairs, reaching a total of 166 observations which were classified according to the observational scale score (Appropriate, Slightly Appropriate, Not Appropriate), which was correlated to the length of time the babies stayed in the neonatal therapy in order to investigate whether there was an association between these variables. Understanding how interactions occur during hospitalization will allow early detection of bonding difficulties and the prevention of infant disorders.

Keywords: Mother-child relations; Child health services; Length of stay.

El hijo recién nacido con dificultades perinatales representa un acontecimiento en extremo movilizador para la madre y la familia en su totalidad. Los primeros encuentros entre la madre y su hijo son fundamentales y fundantes, y delinearán en gran medida el futuro desarrollo del mismo. En la literatura actual no encontramos demasiadas referencias acerca de cómo se desplegarían estos primeros encuentros entre madres y bebés de alto riesgo. Los neonatos de alto riesgo son aquellos que, debido a circunstancias prenatales, perinatales o posnatales, requieren de cuidados especiales, indispensables para lograr su supervivencia así como para disminuir la posibilidad de la aparición de trastornos funcionales graves durante la infancia. Frecuentemente estos recién nacidos deben ser sometidos a diversos tratamientos dependiendo de su patología y/o de la prematurez al momento de nacer (Nikodem, 2009).

Esta condición del recién nacido supone que las primeras interacciones madre-hijo se desarrollarán en un contexto diferente, ya que estos bebés deberán permanecer internados en una Unidad de Cuidados Intensivos Neonatales. En estas condiciones, la díada madre-bebé intentará establecer un vínculo saludable: por su parte, el niño deberá completar su desarrollo fuera del cuerpo de su madre o superar una patología neonatal, mientras que su mamá deberá afrontar no solo la crisis evolutiva que representa el nacimiento, sino también una nueva crisis representada por la internación y la patología del niño (Oiberman, 2005). El contexto específico de la Unidad de Cuidados Intensivos Neonatales delimita un tiempo y un espacio singular dentro del cual se desarrollan los primeros encuentros de la díada, y sin duda imprimirá características particulares a las interacciones.
En este artículo se presentan resultados preliminares de una investigación en la que se indagó acerca de las características de las interacciones madre-bebé de alto riesgo durante la internación en Unidad de Cuidados Intensivos Neonatales; se parte de la hipótesis que el tiempo que el bebé permanece internado en la Unidad de Cuidados Intensivos Neonatales puede ser una variable que incida en la manera en que se desarrollan las primeras interacciones madre-hijo, por lo que se pretendió analizar si los puntajes obtenidos en las interacciones variaban con el transcurso del tiempo de internación. El objetivo del estudio fue entonces describir las interacciones diádicas y conocer si el tiempo de internación incide sobre las mismas. Se considera que este conocimiento sería útil para el equipo de salud, ya que les permitiría mantenerse atentos a estas circunstancias, detectar tempranamente las dificultades e intervenir precozmente evitando que éstas se perpetúen en el tiempo.

\section{Antecedentes: observación de las interacciones madre-bebé de alto riesgo}

La interacción es definida como la conducta manifiesta de la madre y el bebé que se presenta en respuesta y en combinación con la del otro. Se trata de una conducta visible y audible para un tercero (Stern, 1997). En esta investigación se identificaron las interacciones entre la díada, a partir de lo cual y en etapas posteriores se formularán hipótesis respecto del vínculo entre ellos. El concepto de vínculo incluye no solo las conductas observables, sino también la percepción subjetiva de esas conductas por parte de los padres, las fantasías, las tradiciones, los mitos familiares, la historia de cada uno de los miembros de la díada, 
la representación de ese hijo y la representación que la mujer tiene de sí misma como madre (Stern, 1997), entendiendo al vínculo como una ligadura emocional estable característica del mundo mental (Berenstein, 1991). Spitz (1965/2003), por su parte, se refiere al vínculo en término de relaciones de objeto y considera que éstas deben ser satisfactorias tanto para la madre como para el niño y que debe haber interacciones constantes entre los dos participantes, ya que cada uno de ellos provoca las respuestas del otro. El vínculo es bidireccional y recíproco, lo que supone que los padres influyen en las respuestas de los niños, pero éstos también poseen determinadas características, sociales y biológicamente determinadas, que influyen en la conducta de los adultos hacia ellos (Nikodem, 2009). El buen funcionamiento del vínculo madre-bebé es un elemento imprescindible para el desarrollo psíquico y la organización del yo de manera sana y estable ya que, en los momentos iniciales del desarrollo, la psiquis inmadura del lactante depende de un sostén, de la adaptación a sus necesidades provista por la madre o la figura materna (Winnicott, 1971/1987). Bowlby (1969/1976, 1980/1990, 1973/ 1993) propone el concepto de apego, entendido como la relación emocional que el niño establece con sus padres y/o cuidadores, y que le proporciona la seguridad necesaria para un buen despliegue de su personalidad. El estado de seguridad, ansiedad o temor del niño está determinado en gran medida por la accesibilidad y capacidad para responder de la persona con la que se establece el vínculo inicial. Ainsworth (1991) estudió la diferencia en las interacciones madre-hijo y su incidencia en la formación del apego, a partir de lo cual describió tres patrones de apego. Tanto Bowlby como Ainsworth entienden al niño como un participante activo que provoca respuestas en los padres y/o cuidadores.

El término recién nacidos de alto riesgo incluye a bebés prematuros y a término que presentan alguna patología que los hace más vulnerables que el resto de los recién nacidos, por lo que deben permanecer internados en una Unidad de Cuidados Intensivos Neonatales (Nikodem,
2009). Según la Edad Gestacional, tiempo trascurrido entre el primer día del último periodo menstrual hasta el día del parto, los bebés de alto riesgo pueden ser clasificados como prematuros (nacidos antes de las 37 semanas completas de gestación); a término (37-42 semanas de gestación) y postérmino (más de 42 semanas de gestación). Dentro de los recién nacidos prematuros se diferencia a los prematuros moderados, es decir, a los nacidos entre 36 y 32 semanas de Edad Gestacional; los muy prematuros o nacidos entre 31 y 28 semanas de Edad Gestacional; y los prematuros extremos, que son aquellos bebés nacidos antes de las 28 semanas. De acuerdo al Peso de Nacimiento, los bebés pueden ser catalogados como de Peso Adecuado ( $\geq 2,500 \mathrm{~g}$ ), Bajo Peso de Nacimiento ( $\geq 2,500 \mathrm{~g})$, Muy Bajo Peso de Nacimiento ( $\leq 1,500 \mathrm{~g}$ ) y Extremo Bajo Peso de Nacimiento ( $\leq 1,000 \mathrm{~g})$ (Kelly, 2006).

Debido a la condición del bebé al momento de nacer, el contacto entre la madre y éste no es posible de forma inmediata, lo que da lugar a una situación de angustia e incertidumbre (Nikodem, 2009). La madre se contacta con su bebé en el contexto desconocido de la Unidad de Cuidados Intensivos Neonatales, por lo que todos los equipos utilizados en la terapia neonatal son nuevos para ella y pueden asustarla (Nikodem, 2009). Las madres se ven invadidas por múltiples sentimientos, experimentan un fuerte estremecimiento frente al primer encuentro con su bebé en la incubadora, especialmente por su aspecto físico; otras experimentan tristeza, culpa, miedo y extrañeza frente a ese hijo internado (Hauser, Milán, González, \& Oiberman, 2014). La madre puede sentirse ansiosa y con miedo de dañar a su hijo si lo toca, lo que puede llevarla a no visitarlo o no interactuar con él (Nikodem, 2009). A pesar de estas dificultades mencionadas, investigaciones recientes evidencian que, aún en estas condiciones peculiares, es posible mantener un intercambio afectivoconductual amplio aunque con peculiaridades debidas a la situación de internación, donde el niño no está en brazos de la madre sino en una incubadora en la unidad de cuidados intensivos (Santos, 2010). 


\section{Método}

La investigación se desarrolló en el Servicio de Neonatología de una institución pública en la provincia de San Luis, Argentina. Este servicio dispone de una Unidad de Cuidados Intensivos Neonatales con una capacidad aproximada para 20 niños, distribuidos en salas de cuidados intensivos, cuidados intermedios y cuidados medios.

\section{Participantes}

La muestra está formada por 36 díadas madre-bebé de alto riesgo. Se realizó un muestreo aleatorio simple, definiéndose como unidades de análisis a las interacciones madre-bebé de alto riesgo durante la internación. La recolección de datos se realizó durante el año 2012 y 2013, para la cual se solicitó el consentimiento informado a todos los sujetos involucrados, incluyéndose aquellas díadas en las que los bebés eran considerados de alto riesgo y que permanecieron internados en su periodo neonatal en la Unidad de Cuidados Intensivos Neonatales. Respecto de las madres, se seleccionaron madres adolescentes y adultas, que estuvieran solas o con pareja al momento del nacimiento de su hijo.

\section{Instrumentos}

Se utilizó la Escala de observación de vínculo madre-bebé internado en Unidad de Cuidados Intensivos Neonatales (N-EOV-INC) (Santos, 2010) que comprende 6 funciones: función de acercamiento, función contacto con la incubadora, función corporal, función visual, función verbal, función postural.

La función de acercamiento indaga acerca de la posición y distancia que adoptan ambos miembros de la díada para la interacción. La función contacto con la incubadora evalúa si la madre toca o no la incubadora en la que se encuentra su hijo y si introduce las manos en la misma. La función corporal se refiere a conductas de contacto corporal y táctil entre ambos miembros de la díada, e incluye ítems referentes a la ausencia de contacto corporal directo entre la mamá y el bebé así como también conductas de búsqueda de contacto corporal entre ambos. La función visual contempla conductas de contacto visual y de miradas entre ambos miembros de la díada y la distancia entre ambos al realizar las mismas. La función verbal se refiere a conductas verbales, vocales y de sonidos comunicativos, y contiene ítems que discriminan las conductas vocales comunicativas realizadas por la madre y las respuestas del bebé a dichas conductas. La función postural se refiere a la modificación de la postura corporal inicial adoptada en la interacción y la consecuente modificación, o no, de la postura corporal del otro miembro de la díada como respuesta al cambio introducido. Esta función contiene ítems dinámicos de acercamiento y alejamiento para ambos miembros de la díada.

El puntaje obtenido a partir de la suma de todas las funciones se corresponde con un percentil, que permite ubicar a la díada en uno de tres posibles grupos: Situación Vincular No Adecuada, lo que supone que la díada se encuentra en riesgo vincular; Situación Vincular Poco Adecuada; y Situación Vincular Adecuada. El presente instrumento ha superado los estudios de confiabilidad y validez. La N-EOV-INC fue administrada luego de recibir un entrenamiento con la autora.

\section{Procedimientos}

Se realizaron observaciones de las interacciones madre-bebé aplicando la N-EOV-INC durante los momentos en los que la madre ingresaba a la Unidad de Cuidados Intensivos Neonatales para ver a su hijo internado, la primera de ellas durante los primeros 30 días de vida del bebé y continuando con un intervalo de dos o tres días entre las diferentes observaciones a fin de estudiar la evolución de las interacciones. El número de observaciones realizadas varió según cada díada, ya que esto se modificaba en función del tiempo probable de internación de cada recién nacido, que dependía de la gravedad del mismo y de su evolución.

Para el análisis estadístico de los datos obtenidos se utilizó el Paquete Estadístico para 
Ciencias Sociales (SPSS, Statistical Package for the Social Sciences, versión 21) y el software libre $R$ versión 3.1.1. La investigación cuenta con el aval del Comité Académico de la Carrera de Doctorado en Psicología de la Facultad de Psicología de la Universidad, que certifica que la investigación cumple con los requisitos éticos.

\section{Resultados}

La descripción socio-demográfica de la muestra se presenta en la Tabla 1.

\section{Interacciones madre-bebé en la Unidad de Cuidados Intensivos Neonatales}

Se realizaron 166 observaciones de las interacciones de 36 díadas madre-bebé de alto riesgo durante el periodo de internación del bebé en la Unidad de Cuidados Intensivos Neonatales. A partir del puntaje obtenido mediante la aplicación de la N-EOV-INC se pudo clasificar la situación

Tabla 1

Descripción socio-demográfica de la muestra $(N=36)$

\begin{tabular}{|c|c|c|}
\hline Descripción socio-demográfica & $\mathrm{n}$ & $\%$ \\
\hline \multicolumn{3}{|l|}{ Edad materna } \\
\hline Menores de 18 años & 5 & 13,9 \\
\hline Entre 19 y 24 años & 21 & 58,3 \\
\hline Entre 25 y 30 años & 6 & 16,6 \\
\hline Mayores de 31 años & 4 & 11,1 \\
\hline \multicolumn{3}{|l|}{ Nivel educativo materno } \\
\hline Estudios primarios incompletos & 2 & 5,6 \\
\hline Estudios primarios completos & 17 & 47,2 \\
\hline Estudios secundarios completos & 15 & 41,6 \\
\hline Estudios terciarios/universitarios completos & 2 & 5,6 \\
\hline \multicolumn{3}{|l|}{ Clasificación del bebé según edad gestacional } \\
\hline Recién nacidos a término & 4 & 11,1 \\
\hline Prematuros & 32 & 88,9 \\
\hline \multicolumn{3}{|l|}{ Clasificación del bebé según peso de nacimiento } \\
\hline Peso Adecuado & 4 & 11,1 \\
\hline Bajo Peso de Nacimiento & 14 & 38,9 \\
\hline Muy Bajo Peso de Nacimiento & 16 & 44,4 \\
\hline Extremo Bajo Peso de Nacimiento & 2 & 5,6 \\
\hline \multicolumn{3}{|l|}{ Diagnóstico del recién nacido } \\
\hline Enfermedad respiratoria & 20 & 55,5 \\
\hline Retardo en el crecimiento intrauterino & 10 & 27,7 \\
\hline Otras (sepsis, intestinal, genética, neurológica, ictericia) & 6 & 16,8 \\
\hline
\end{tabular}

vincular de la díada en cada observación según los tres grupos propuestos por el instrumento: Situación Vincular No Adecuada (puntajes entre 2 y 8), Situación Vincular Poco Adecuada (puntaje de 9) y Situación Vincular Adecuada (puntajes entre $10 \mathrm{y}$ 16). En la Tabla 2 se presentan las características de cada grupo respecto de la posición adoptada para la interacción, la cercanía y el contacto con la incubadora por parte de la madre y la modificación postural de ambos miembros de la díada.

Se obtuvo que, en 102 observaciones, la Situación Vincular de las díadas fue Adecuada. Dentro de este grupo se encontraron diferencias en las interacciones corporales, verbales y visuales, lo que dio lugar a la construcción de dos subgrupos no contemplados en la versión original del instrumento. Un primer subgrupo se configuró con 43 observaciones, donde las interacciones fueron exitosas a nivel corporal, verbal y visual. En estas observaciones, el puntaje predominante fue 14 puntos (32,5\%). En un segundo subgrupo formado por 59 observaciones con una Situación Vincular Adecuada se observó que las díadas no lograban interacciones exitosas en las tres áreas arriba descriptas, ya sea por la ausencia de propuesta de interacción o de respuesta de un miembro. En 37 observaciones de este subgrupo existieron dificultades en las interacciones visuales, predominando el puntaje $10(62,7 \%$ de las observaciones); en 15 observaciones hubo dificultades en las interacciones verbales, predominando el puntaje $12(25,4 \%)$, y en 7 observaciones hubo dificultades en las interacciones corporales, predominando el puntaje 11 (11,9\% de las observaciones).

Por otro lado, en 25 observaciones se encontró que la Situación Vincular fue Poco Adecuada. Las interacciones corporales fueron exitosas en 23 observaciones. En las interacciones visuales hubo mayores dificultades, siempre que las madres miraron a sus hijos éstos no respondieron, y cuando en dos situaciones los bebés dirigieron la mirada hacia sus madres éstas tampoco respondieron. Las interacciones verbales fueron escasas: las madres se dirigieron verbalmente a sus hijos en 11 observaciones; sin embargo, los bebés respondieron 
Tabla 2

Características de las interacciones: posición, acercamiento, contacto con la incubadora, modificación postural ( $N=36)$

\begin{tabular}{|c|c|}
\hline Situación vincular & Características de la interacción \\
\hline Situación vincular adecuada & 102 observaciones \\
\hline Posición & 102 cercana a la incubadora \\
\hline Acercamiento & 97 de frente al bebé - 5 de espalda \\
\hline Contacto con la incubadora & 97 introduce las manos \\
\hline \multirow[t]{4}{*}{ Modificación postural } & 86 no hay modificación \\
\hline & 4 madre se aleja - bebé acepta \\
\hline & 10 madre se acerca - bebé acepta \\
\hline & 2 bebé se acerca - mamá acepta \\
\hline Situación vincular poco adecuada & 25 observaciones \\
\hline Posición & 25 cercana a la incubadora \\
\hline Acercamiento & 18 de frente al bebé - 7 de espalda \\
\hline Contacto con la incubadora & 25 introduce las manos \\
\hline \multirow[t]{3}{*}{ Modificación postural } & 22 no hay modificación \\
\hline & 2 madre se aleja - bebé acepta \\
\hline & 1 madre se acerca - bebé acepta \\
\hline Situación vincular no adecuada & 39 observaciones \\
\hline Posición & 37 cercana a la incubadora - 2 alejada \\
\hline Acercamiento & 29 de frente al bebé - 10 de espalda \\
\hline Contacto con la incubadora & 35 introduce manos - 4 no introduce manos \\
\hline \multirow[t]{3}{*}{ Modificación postural } & 31 no hay modificación \\
\hline & 8 madre se aleja - 7 bebé acepta \\
\hline & -1 bebé se aleja \\
\hline
\end{tabular}

al estímulo verbal solo en 4 de las mismas. En este grupo de observaciones Poco Adecuadas el puntaje predominante fue 9 .

Finalmente, en 39 observaciones la Situación Vincular de las díadas fue clasificada como No Adecuada. Se distinguieron dos subgrupos; en el primero, formado por 21 observaciones, no hubo interacciones exitosas a nivel corporal, visual ni verbal. En la totalidad de las observaciones las díadas no buscaron establecer contacto corporal; solo en una observación el bebé buscó tocar a su madre, sin obtener respuesta por parte de ella. En cuanto a los intercambios visuales, las madres buscaron visualmente a sus hijos en 17 observaciones sin obtener alguna respuesta de parte de los bebés. Solo en una observación, el bebé buscó con la mirada a su madre pero ésta no respondió. A nivel verbal, estas madres no les hablaron a sus hijos en 20 observaciones, mientras que solo una madre lo hizo pero su bebé no respondió al estímulo. En este subgrupo predominó el puntaje 7 (33\% de las observaciones). El segundo subgrupo está representado por 18 observaciones. Las interacciones corporales fueron más exitosas: todas las madres tocaron a los bebés, y ellos respondieron aceptando el contacto en 17 observaciones; 2 bebés buscaron tocar a sus madres, a lo que una respondió de manera positiva y la otra no. A nivel visual, en 17 observaciones las madres miraron a sus hijos pero no obtuvieron respuestas por parte de ellos. Finalmente en cuanto a los intercambios verbales, en 15 observaciones las madres no le hablaron a sus hijos, mientras que en 5 observaciones lo hicieron pero solo en una el bebé respondió al estímulo verbal. En este subgrupo predominó el puntaje 8 (71\% de las observaciones).

En las observaciones se relacionó la edad de las madres con la situación vincular. Los grupos etarios en los que tuvieron mayor presencia los puntajes no adecuados y poco adecuados fueron entre $19-24$ años (49,6\% de las observaciones) y mayores de 31 años (50\% de las observaciones). El 
coeficiente de correlación de Pearson no arrojó una asociación significativa entre la edad materna y el puntaje obtenido en la interacción.

\section{Relación entre las interacciones madre-bebé y el tiempo de internación en la terapia neonatal}

A fin de estudiar si el transcurso del tiempo de internación incidía sobre las interacciones madrebebé se realizó un análisis de varianza de un factor con medidas repetidas. Este análisis consistió en medir el puntaje de las interacciones en diferentes momentos de tiempo (número de observación). El orden creciente de las observaciones (observación 1, observación $2 \ldots$ ) implica un incremento del tiempo transcurrido en la Unidad de Cuidados Intensivos Neonatales, por lo que se hipotetizó que los puntajes de las interacciones podrían variar de una a otra medición. Para llevar a cabo este análisis se incluyeron las díadas con mayor cantidad de observaciones; esta selección se realizó a partir del cálculo del valor medio de observaciones por díada $(M=4,6)$, escogiendo aquellas que contaban con un número de observaciones cercano a la media; es decir, 5 o más. De la muestra total se escogió una submuestra de 16 díadas para analizar la incidencia del tiempo de internación en las interacciones observadas. El análisis se redujo a las primeras cinco observaciones presentes en todas las díadas de la submuestra, realizándose finalmente sobre 104 observaciones ( $62 \%$ de la muestra total).

El periodo total de internación difiere en cada caso. De los 16 bebés seleccionados para este análisis, 4 permanecieron internados de 61-70 días; 3 bebés más de 71 días y 3 bebés estuvieron internados entre 41-50 días. En menor medida, 2 bebés permanecieron en la incubadora por un tiempo de 31-40 días y otros 2 bebés entre 51-60 días. Finalmente, en un caso la internación duró entre 11-20 días y en otro entre 21-30 días. Al momento de la primera observación, los bebés en promedio habían permanecido internados por 8 días; en la segunda observación durante 13 días, en la tercera durante 18 días, 24 días en la cuarta observación y finalmente un promedio de 27 días en la quinta observación.
Los resultados señalan que el transcurso del tiempo en internación no tendría una incidencia significativa sobre los puntajes obtenidos en las interacciones diádicas. En las cinco mediciones el valor de los estadísticos multivariados es de 0,870, siendo superior al nivel crítico (Nivel de significación - Sig $=0,05$ ); esto supone que no hay diferencias relevantes entre las medias en cada punto de tiempo $(M=10,13 ; 10,81 ; 10,88 ; 10,38 ; 10,75)$. En la Figura 1 se visualiza que en la primera observación las díadas obtuvieron los puntajes más bajos; esto cambia en la segunda observación, donde se presenta un incremento pronunciado en los puntajes obtenidos, para continuar incrementándose de manera menos marcada en la tercera observación. Sin embargo, en la cuarta observación se produce una disminución de los puntajes de las interacciones, aunque sin llegar a ser tan bajos como en la primera, para finalmente volver a aumentar en la quinta observación. Esta figura permite observar que, si bien la media de los puntajes interactivos varía levemente con el transcurso del tiempo de internación entre el valor 10 y 11, no se puede afirmar que el tiempo incida significativamente en los mismos de manera negativa o positiva.

\section{Discusión}

El nacimiento de un bebé que requiere de su internación en una Unidad de Cuidados

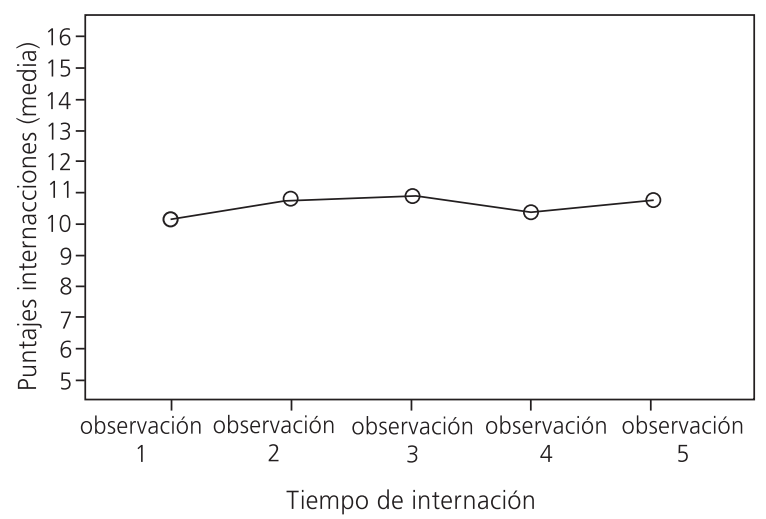

Figura 1. Incidencia del tiempo de internación en las interacciones diádicas $(\mathrm{N}=16)$. 
Intensivos Neonatales supone una experiencia inédita. Las madres pueden sentir que su rol y sus funciones han sido ocupados por los profesionales de la salud y los elementos técnicos; que esos recién nacidos no les pertenecen, sino que son "hijos de las máquinas" y por lo tanto deberán competir con ellas (Fava Vizzielo, Zorzi, \& Bottos, 1993). A pesar de ser influidas por el ambiente de la internación, las interacciones entre la madre y su bebé en la Unidad de Cuidados Intensivos Neonatales podrán dar lugar a un intercambio afectivo y conductual amplio y enriquecedor, como sostiene Santos (2010). Los resultados de la presente investigación coinciden con esta afirmación, ya que claramente la gran mayoría de las díadas lograron interacciones satisfactorias, con cualidades específicas y diferentes (Situación Vincular Adecuada - 61\%); mientras que, en menor medida, las interacciones fueron Poco Adecuadas (14\%) y No Adecuadas (25\%). En este sentido, que las interacciones sean exitosas o con dificultades, se verá determinado en cierto modo por cómo los padres han vivido la prematurez y/o la patología, así como la experiencia de la internación. Un elemento que ha sido asociado a dificultades en las interacciones y en el desarrollo de los bebés de alto riesgo es la menor edad materna (Marotta, 2007). En la presente investigación no se encontró una relación significativa entre este factor y las características de las interacciones diádicas; por el contrario, se observa que los puntajes no adecuados y poco adecuados se distribuyen en grupos etarios bien variados.

Los resultados de esta investigación sugieren que el transcurso del tiempo de internación no incidiría en la cualidad de las interacciones diádicas, al menos en las 16 díadas analizadas. A pesar de que se visualiza una variación de los puntajes a medida que transcurren las observaciones, ésta no es significativa, ya que el valor medio entre 10 y 11 continúa siendo adecuado. Esta leve fluctuación de los puntajes interactivos parecería deberse a las características mismas de la vinculación, que no sigue un patrón definido de manera rígida e irreversible, sino que, por el contrario, se trata de un proceso dinámico en constante movimiento, que sin duda se ve atravesado e influido por los diferentes elementos propios del contexto de la internación, entre los que podemos mencionar el delicado estado emocional de la madre, lo impredecible de la condición clínica del bebé y el apoyo familiar recibido, entre otros. Brazelton y Cramer (1993) conciben a las interacciones como un proceso; es decir, que pueden haber momentos de participación y de cese de la participación por parte de ambos miembros de la díada. Sin lugar a dudas, esta concepción puede aplicarse al seguimiento de las interacciones de madres y bebés durante la internación, donde se presentan estos diferentes momentos de encuentro y desencuentro. Quizás la tarea decisiva sea poder decodificar cuáles son los momentos que prevalecen y la influencia que éstos pueden tener en el desarrollo del vínculo temprano.

Cuando este proceso de vinculación tiene lugar durante la internación, las madres deberán "adoptar" a esos hijos, apropiarse de sus funciones maternas y, para eso, probablemente algunas necesiten más tiempo que otras. Los resultados de la presente investigación muestran que, en la primera observación, los puntajes obtenidos en las interacciones son relativamente más bajos, lo que podría deberse a la necesidad de las díadas de adecuarse al contexto de la Unidad de Cuidados Intensivos Neonatales: la madre debe llevar a cabo el duelo por el hijo imaginado y empezar a contactarse con el hijo real; el bebé, por su parte, debe alcanzar un mayor estado de alerta y actividad. Las interacciones mejoran sutilmente en la segunda y tercera observación para disminuir nuevamente el puntaje en la cuarta, alrededor de los 24 días de internación. Se puede hipotetizar que, en este momento cercano al mes de hospitalización, las díadas comienzan a resentirse frente al stress y el gasto de energía psíquica que supone la internación, las presiones y exigencias del ambiente médico, el alejamiento familiar y las dificultades económicas y sociales profundizadas por la prolongada internación. Las díadas parecen reacomodarse frente a esta situación en la quinta observación, ya que las interacciones mejoran levemente. Este proceso de acomodación de las interacciones 
permite suponer que los cambios en las mismas no remitirían tanto al tiempo de internación como sí a sus cualidades intrínsecas. Fogel, Garvey, Hsu, y West-Stroming (2006) afirman que las relaciones cambian debido a su variabilidad intrínseca, la cual no puede ser ignorada o considerada un error en las mediciones. Por lo general, las díadas se involucran en un patrón de conducta y, a la vez, eventualmente este patrón finaliza y otro diferente comienza dentro de una secuencia temporal. Las diferencias entre las diadas se pueden explicar a partir de la utilización de patrones distintos y por ende, de las acciones disimiles llevadas a cabos dentro de ellos. Si bien en una diada ambos participantes se encuentran involucrados en el mismo patrón, hay lugar para la novedad, para que nuevos patrones emerjan espontáneamente y se produzcan los cambios en las relaciones.

La descripción de las interacciones madrebebé presentada en esta investigación puede contribuir a futuras modificaciones del instrumento elaborado por Santos (2010), donde las interacciones pueden clasificarse en función de los puntajes obtenidos en No Adecuadas (2-8 puntos), Poco Adecuadas (9 puntos) y Adecuadas (10-16 puntos). Sin embargo, en la presente investigación se notó que esta discriminación no permite visualizar las sutiles diferencias que pueden presentarse dentro de cada una de estas situaciones vinculares, lo que se debería a que el rango de puntajes es demasiado amplio en los dos extremos. A partir de los resultados obtenidos se construyeron subgrupos dentro de la situación vincular adecuada y no adecuada en base a las diferencias que se presentaron en las interacciones. En la Situación Vincular Adecuada resultaron cuatro subgrupos: aquel en el que se presentaron interacciones exitosas en el área corporal, visual y verbal (puntajes entre 12-16, predominando el 14); aquel en el cual hubo interacciones exitosas pero las dificultades estuvieron en las interacciones verbales (puntajes 10- 14 predominando el puntaje 12); otro en el que las dificultades se presentaron en los intercambios visuales (puntajes entre 10-13, predominando el 10) y finalmente un subgrupo en el que hubo intercambios entre las díadas pero las dificultades estuvieron en los contactos corporales (puntaje entre 10-12, prevaleciendo el 11). En el grupo Situación Vincular No Adecuada se diferenciaron dos subgrupos: aquel en el que no hubo interacciones exitosas en ninguna de las tres áreas (predominando el puntaje 7) y otro subgrupo donde existieron mayores interacciones pero aún con dificultades (predominando el puntaje 8).

La presente investigación posee la limitación de contar con una muestra pequeña para el análisis de la incidencia del tiempo de internación. Asimismo, no se pudo entrar en diálogo con resultados de otros estudios acerca de la asociación entre las interacciones madre-bebé y el tiempo de internación. Esto se debe a la escasez de estudios sobre la temática, por lo que será fundamental considerar estos aspectos en posteriores investigaciones.

\section{Consideraciones Finales}

Estudiar el modo en que el tiempo de internación puede influir en los intercambios entre madres e hijos constituye un elemento relevante en lo que a políticas de salud se refiere. La vigilancia atenta de las interacciones permitiría detectar las fallas que pueden presentarse en la adaptación madre-bebé o a la situación de internación así como los signos de ansiedad, depresión o rechazo en las madres, constituyendo una oportunidad única para evitar que estos problemas redunden en trastornos futuros en la infancia. Conocer las características que pueden adoptar las interacciones madre-bebé en Unidad de Cuidados Intensivos Neonatales así como los factores que pueden incidir sobre ellas contribuiría a la tarea de los profesionales de salud, ya que podrían fortalecer los intercambios positivos madre-hijo y propiciar desde la institución una mejor comunicación en las díadas que presentan mayores dificultades.

\section{Colaboradores}

M.P. HAUSER participó en el desarrollo de la estrategia experimental, la recopilación de datos, 
tabulación y análisis de los resultados y la preparación del artículo. T.A. MILÁN, M.C. GONZÁLEZ, y A. OIBERMAN participaron en el desarrollo de la estrategia experimental, análisis de los resultados y la preparación del artículo.

\section{Referencias}

Ainsworth, M. D. S. (1991). Attachments and other affectional bonds across the life cycle. In C. M. Parkes, J. Stevenson-Hinde, \& P. Marris (Eds.), Attachment across the life cycle (pp.33-51). London: Routledge.

Berenstein, I. (1991). Reconsideración del concepto de vínculo. Psicoanálisis, 13(2), 219-235.

Bowlby, J. (1976). El apego. Barcelona: Paidós Ibérica. (Trabajo originalmente publicado en 1969).

Bowlby, J. (1990). La pérdida. Barcelona: Paidós Ibérica. (Trabajo originalmente publicado en 1980).

Bowlby, J. (1993). La separación. Barcelona: Paidós Ibérica. (Trabajo originalmente publicado en 1973).

Brazelton, T. B., \& Cramer, B. G. (1993). La relación más temprana: Padres, bebés y el drama del apego inicial. Barcelona: Paidós Ibérica.

Fava Vizziello, G., Zorzi, C., \& Bottos, M. (Comp.). (1993). Los hijos de las máquinas: La vida de los niños internados en terapias intensivas neonatales. Buenos Aires: Nueva Visión.

Fogel, A., Garvey, A., Hsu, H., \& West-Stroming, D. (2006). Change processes in relationships: A relationalhistorical research approach. New York: Cambridge University Press.
Hauser, M. P., Milán, T. A., González, M. C., \& Oiberman, A. (2014). Impacto emocional en madres de bebés internados en una unidad de cuidados intensivos neonatales. Aplicación de la entrevista psicológica perinatal. Investigaciones en Psicología, 19(1), 63-83.

Kelly, M. M. (2006). The basics of prematurity. Journal of Pediatric Health Care, 20(4), 238-244.

Marotta, C. (2007). Un estudio cualitativo del vínculo madre-hijo en adolescentes madres de sectores de pobreza. Revista Uruguaya de Enfermería, 2(2), 11-25.

Nikodem, M. R. (2009). Niños de alto riesgo: Intervenciones tempranas en el desarrollo y la salud infantil. Buenos Aires: Paidós Ibérica.

Oiberman, A. (2005). Nacer y después... aportes a la psicología perinatal. Buenos Aires: JCE Ediciones.

Santos, M. S. (Mayo, 2010). Psychoneonatology: Results of a new observation scale of the bond mother-baby in incubator. Paper presented at the XXII European Congress Perinatal Medicine, Granada, Spain.

Spitz, R. (2003). El primer año de vida del niño $\left(20^{a}\right.$ reimp.). México: Fondo de Cultura Económica. (Trabajo originalmente publicado en 1965).

Stern, D. (1997). La constelación maternal: La psicoterapia en las relaciones entre padres e hijos. Barcelona: Paidós Ibérica.

Winnicott, D. (1987). Realidad y juego. Barcelona: Gedisa. (Trabajo originalmente publicado en 1971).

Recibido: Noviembre 25, 2014

Versión final: Junio 1, 2015

Aprobado: Agosto 25, 2015 